\title{
Détermination de l'adhérence des mortiers et autres revêtements de surface
}

\section{SOMMAIRE}

1. Objet

2. Eprouvettes

2.1. Échantillon de la gâchée de fabrication.

2.2. Description détaillée des éprouvettes.

2.3. Nombre d'éprouvettes.

2.4. Application du revêtement.

\section{Matériel}

3.1. Matériel d'essais.

3.2. Machine à découper le revêtement de surface.

3.3. Matériel de pesée.

3.4. Matériel d'étuvage.

4. Conduite de l'essai

5. Résultats de l'essai

6. Procès verbal d'essai

Annexe : Essais sur murs in situ.

\section{OBJET}

La présente recommandation décrit une méthode de détermination de l'adhérence des mortiers et autres revêtements de surface sur le béton cellulaire autoclavé.

L'adhérence est définie par la force de traction requise pour arracher une section circulaire ou carrée de mortier ou de revêtement de surface dont le périmètre a été préalablement découpé jusqu'au support.

\section{EPROUVETTES}

\section{1. Échantillon de la gâchée de fabrication}

Les éprouvettes en béton cellulaire autoclavé doiveni être prélevées au hasard dans une gâchée de fabrication du produit à tester. On doit laisser les éprouvettes refroidir, enfermées dans un sac étanche, afin de prévenir une perte d'humidité, pendant une durée minimale de 24 heures avant application du revêtement de surface.

\subsection{Description détaillée des éprouvettes}

On utilise des éprouvettes prismatiques de dimensions minimales $350 \times 200 \mathrm{~mm}$ et d'épaisseur minimale $75 \mathrm{~mm}$. La surface recevant le revêtement devra être identique à celle du produit fabriqué.

\subsection{Nombre d'éprouvettes}

L'ensemble soumis à l'essai doit comprendre trois éprouvettes pour chaque type de revêtement à tester. En plus, on utilise deux éprouvettes pour déterminer la masse volumique sèche de chaque gâchée de fabrication.

\subsection{Application du revêtement}

Enduit ou plâtre doivent être appliqués aux éprouvettes placées à l'intérieur d'un coffrage et les dimensions du coffrage ajustées pour donner l'épaisseur voulue au revêtement. Les procédés d'application doivent être strictement conformes aux recommandations des fabricants.

Chaque éprouvette doit recevoir le revêtement de surface à tester sur une face. Le revêtement peut être appliqué sur la face de l'éprouvette se trouvant soit en position horizontale soit en position verticale.

Les revêtements à base de ciment doivent être appliqués après 1 heure et demie de malaxage et doivent être portés a maturation, bien protégés pendant une période de 28 jours avant l'essai. Les autres revêtements doivent être portés à maturation suivant les recommandations des fabricants.

\section{MATERIEL}

\subsection{Matériel d'essai}

La machine d'essai, qui peut être utilisée manuellement, doit être capable d'appliquer et de mesurer une force de traction perpendiculaire au plan du revêtement par l'intermédiaire d'un disque ou d'un carré d'application de charge maintenu dans la partie soumise à l'essai par un adhésif.

Les disques ou carrés d'application de charge doivent être de diamètre ou de côté de 50 ou $80 \mathrm{~mm}$.

\subsection{Machine à découper le revêtement de surface}

Quand il s'agit de tester des sections circulaires, on utilise un carottier capable de couper à travers le revêtement de surface pour donner des sections cylindriques de 50 ou $80 \mathrm{~mm}$ de diamètre et d'épaisseur allant jusqu'à $50 \mathrm{~mm}$.

Quand il s'agit de tester des sections carrées on utilise un banc de scie à disque.

\subsection{Matériel de pesée}

Une balance ou un dynamomètre de précision $0,1 \%$.

\subsection{Matériel d'étuvage}

On utilise une étuve qui doit être régulée à une température de $105 \pm 5^{\circ} \mathrm{C}$ pour déterminer la masse volumique sèche. 